\title{
USO DE INMUNOGLOBULINAS INTRAVENOSAS EN UNA PACIENTE OBSTÉTRICA CON CHOQUE SÉPTICO: REPORTE DE CASO Y REVISIÓN DE LA LITERATURA
}

\author{
Use of intravenous immunoglobullins in an \\ obstetric patient with septic shock: Case report \\ and review of the literature \\ Olga Lucía Cuero-Vidal, $\mathrm{MD}^{1}$; Diego Fernando Moreno-Sánchez, $\mathrm{MD}^{2}$; \\ María del Mar Torres-Bejarano, MD ${ }^{3}$; Johana Alejandra Moreno-Drada, $\mathrm{MSc}^{4}$ \\ Recibido: noviembre 13/15 - Aceptado: octubre 26/16
}

\section{RESUMEN}

Objetivo: reportar el caso de una paciente obstétrica que desarrolló un shock séptico secundario a una infección gastrointestinal y el uso de inmunoglobulina endovenosa (IVIG) como terapia coadyuvante y hacer una revisión de la literatura publicada del uso de inmunoglobulinas en shock séptico con énfasis en su uso en el embarazo.

Materiales y métodos: se presenta el caso de una paciente con embarazo de 36 semanas, quien presentó un choque séptico secundario a una infección

1 Médico especialista en Obstetricia y Ginecología; magíster en Epidemiología, Servicio de Obstetricia y Ginecología, Asistencial Hospital San Juan de Dios. Docente Universidad Javeriana Cali, Grupo Investigación Integral Médica (GIIM), Cali (Colombia). linary67@hotmail.com

2 Médico especialista en Medicina Interna; especialista en Medicina crítica y Cuidado intensivo; magíster en Epidemiología, Servicio de Medicina Interna, Asistencial Hospital San Juan de Dios, Asistencial Clínica de los Remedios. Docente Universidad del Valle; docente Universidad Javeriana, Grupo Investigación Integral Médica (GIIM), Cali (Colombia).

3 Médico especialista en Obstetricia y Ginecología, Servicio de Obstetricia y Ginecología, Asistencial Hospital San Juan de Dios. Docente Universidad Javeriana, Cali, Grupo Investigación Integral Médica (GIIM), Cali (Colombia).

4 Odontóloga. Maestría en Epidemiología, Servicio de Odontología y Cirugía Maxilo Facial, Asistencial Hospital San Juan de Dios, Grupo Investigación Integral Médica (GIIM), Cali (Colombia). gastrointestinal y progresó con falla orgánica multisistémica. En vista de la pobre respuesta al tratamiento instaurado se inició terapia coadyuvante con inmunoglobulina enriquecida en IgM con una buena respuesta. Se realizó una búsqueda de la literatura en la bases de datos de Medline vía PubMed, Direct Sciencie, Biblioteca Cochrane y SciELO utilizando las palabras clave de los Descriptores en Ciencias de la Salud (DeCS) como inmunoglobulina endovenosa y sepsis en embarazo.

Resultados: se encontraron 6 artículos relacionados directamente con el uso de IVIG en sepsis en el embarazo, 1 revisión de tema, 2 reportes de caso y 3 guías diagnósticas y de manejo. Las inmunoglobulinas intravenosas se han utilizado con mayor frecuencia en pacientes no obstétricas en shock séptico como terapia coadyuvante especialmente en infección por estreptococos y estafilococos.

Conclusión: las inmunoglobulinas intravenosas podrían ser una alternativa como coadyuvante en manejo de shock séptico en gestantes con pobre respuesta al tratamiento convencional. Se necesitan estudios analíticos que incluyan gestantes para confirmar estos hallazgos. 
Palabras clave: sepsis, inmunoglobulinas intravenosas, choque séptico.

\section{ABSTRACT}

Objective: To report the case of an obstetric patient who developed septic shock secondary to a gastrointestinal infection, and the use of intravenous immunoglobulin (IVIG) as an adjunct, and to conduct a review of the published literature on the use of immunoglobulins in septic shock with emphasis on its use during pregnancy.

Materials and methods: Case report of a patient with 36 weeks of gestation who presented with septic shock secondary to a gastrointestinal infection resulting in multiple organ failure. In view of poor response to treatment, adjunctive therapy with IgM-enriched immunoglobulin therapy was initiated, achieving good response. A search of the literature was conducted in the Medline, Direct Science, Cochrane Library and SciELO databases using Health Science Descriptors (DeCS) such as intravenous immunoglobulin and sepsis in pregnancy.

Results: Six articles directly related to the use of IVIG in sepsis during pregnancy were found, including 1 review, 2 case reports and 3 diagnosis and management guidelines. Intravenous immunoglobulins have been used with greater frequency in non-obstetric patients in septic shock as an adjunct especially in streptococcal and staphylococcal infections.

Conclusion: Intravenous immunoglobulins could be an alternative as adjunct therapy in the management of septic shock in pregnant women who respond poorly to conventional treatment. Analytical studies including pregnant women are needed in order to confirm these findings.

Key words: Sepsis, intravenous immunoglobulins, septic shock.

\section{INTRODUCCIÓN}

La sepsis es definida como una infección con manifestaciones sistémicas y se considera severa cuando se presenta disfunción orgánica o hipoperfusión tisular, la cual progresa a un shock séptico cuando hay la persistencia de hipotensión a pesar de la adecuada reposición de líquidos endovenoso (1-3); estas definiciones se desarrollaron a partir de pacientes no obstétricas, pero se aplican igual durante la gestación $(4,5)$. La incidencia del shock séptico en gestantes se reporta en 2,3 por 100.000 embarazos (4), se ha descrito que se presenta más frecuentemente en el posparto (46\%) que durante el embarazo (44\%) y el parto (10\%) (5). La sepsis en la gestante es la tercera causa de mortalidad materna a nivel mundial (6) y es una de las principales causas de admisión a las unidades de cuidado intensivo (7). Aunque la mortalidad materna por sepsis ha descendido del 11,6 en 1990, a 9,1\% en 2013, sigue afectando principalmente a mujeres de países en desarrollo, donde se presentan cerca del $99 \%$ de las muertes maternas esta causa (8). En países desarrollados la tasa de mortalidad por sepsis en el embarazo es cercana al $4 \%$, mientras que en los países en vía de desarrollo es del 10,4\% (6). En Bogotá (Colombia) se reporta que en el 2103 el total de muertes maternas por sepsis obstétrica fue del 14,2\% (9).

El embarazo representa una situación especial por los cambios fisiológicos que ocurren por acción de los estrógenos, las prostaciclinas y el óxido nítrico que puede enmascarar las fases iniciales de la sepsis que, si no son reconocidas a tiempo, llevan al deterioro progresivo de la materna $(4,10,11)$. El embarazo, por ser un estado de alcalosis respiratoria compensado con una leve acidosis metabólica, lleva a que la mujer gestante, ante un cuadro séptico, tenga pocas reservas para combatir la acidosis metabólica desencadenada por la hipoperfusión tisular que acompaña la sepsis, hecho que debe ser tenido en cuenta cuando se da tratamiento a una gestante en esta condición; además, se debe tener considerar que la disminución de la creatinina y el nitrógeno ureico en el embarazo puede dificultar la adecuada interpretación de los resultados en pacientes con sepsis (10-12). Por otra 
parte, la mujer embarazada hace algunos ajustes inmunológicos para tolerar el embarazo tales como alteraciones de human leukocyte antigen (HLA clase I en el trofoblasto, síntesis de citoquinas inmunosupresoras, expresión de proteína del complemento, y cambios en la subpoblación de leucocitos tales como reducción de los linfocitos T, incremento de los macrófagos y las células natural killer, aunque es importante resaltar que el embarazo no se considera un estado inmunosupresor, sino un estado de inmunotolerancia $(10,13)$.

El diagnóstico de la sepsis en el embarazo se basa en el reconocimiento de alguno de los signos de sepsis tales como pirexia, hipotermia, taquicardia, hipoxia, oliguria, hipotensión, deterioro de la conciencia, no respuesta al tratamiento y la sospecha de un foco séptico (14).

El manejo y tratamiento en general de la sepsis en la embarazada son similares a los de la no embarazada, iniciando con una adecuada resucitación con líquidos, evaluación de la perfusión de órganos y el uso de antibiótico de amplio espectro según el protocolo de la institución y la erradicación quirúrgica del foco infeccioso, previa toma de cultivos y monitorización fetal (15). El temprano reconocimiento y manejo adecuado de la sepsis en el embarazo determinarán el resultado materno fetal $(7,14)$. Por otra parte, para el manejo de la sepsis y en especial del shock séptico se ha descrito el uso de inmunoglobulinas, las cuales actuarían neutralizando las endotoxinas y exotoxinas bacterianas (16), además modularían la respuesta del huésped a la infección limitando la acción de las citoquinas y la activación de cascada del complemento; sin embargo, su uso es motivo de controversia aunque se ha descrito que se debe considerar la utilización de inmunoglobulinas intravenosas (IVIG) como terapia coadyuvante en el tratamiento del shock séptico, especialmente cuando hay una pobre respuesta a las medidas convencionales (17-19). En la literatura internacional hay pocas menciones a su uso en pacientes obstétricas.
El objetivo de este estudio es presentar el caso de una gestante quien desarrolló shock séptico por E. Coli secundaria a infección gastrointestinal que progresó a una falla orgánica multisistémica, y debido a la pobre respuesta al tratamiento convencional de la sepsis recibió terapia coadyuvante con inmunoglobulina endovenosa, y hacer una revisión del uso de inmunoglobulinas en shock séptico con énfasis en su uso en el embarazo.

\section{PRESENTACIÓN DEL CASO}

Paciente de 29 años de edad, mestiza, ama de casa, G1P0 con una edad gestacional de 36 semanas por ecografía, quien ingresa el 19 de mayo de 2015 a un hospital de segundo nivel, de una cuidad intermedia del departamento de Valle del Cauca (Colombia), con un cuadro clínico de escalofríos, temperatura de 39 ${ }^{\circ} \mathrm{C}$, dolor lumbar y deposiciones diarreicas en 3 episodios, percibía movimientos fetales y tenía controles prenatales normales. Hemograma al ingreso informa 3000 leucocitos, hemoglobina $11 \mathrm{mg} / \mathrm{dl}$ y plaquetas de 135.000; 7 horas después se torna disneica, con dolor abdominal y disminución de movimientos fetales. Por tal razón, es remitida el 20 de mayo a una institución de alta complejidad, centro de referencia de pacientes del sur-occidente colombiano, ubicada en Cali, que atiende pacientes afiliadas a los regímenes subsidiado por el Estado y contributivo del sistema de seguridad social en Colombia. Allí se recibe la paciente en regular condición, consciente, orientada, disneica, con una frecuencia respiratoria (FR) de 28 por minuto y taquicardia (frecuencia cardiaca de 110 latidos por minuto), con dolor en hemiabdomen superior. Se toma de nuevo hemograma que reporta 26.000 leucocitos, bandas $8 \%$, plaquetas 108.000. Dos horas después de su ingreso su estado se deteriora, se encuentra hipotensa $(80 / 50 \mathrm{~mm} / \mathrm{hg})$ y con acidosis metabólica severa, lactoacidemia de $8,8 \mathrm{mmol} / \mathrm{L}$; se le realiza una monitorización fetal que muestra desaceleraciones variables de la frecuencia cardiaca fetal, por lo cual se decide terminar el embarazo por cesárea, y a las 
6:45 a.m. nace un feto masculino con apgar 8/10 y peso de 2800 g. La paciente presenta atonía uterina, por lo cual se le realiza plicatura según técnica de B-Lynch, y durante el intraoperatorio presenta paro cardiorrespiratorio que requiere reanimación cardiopulmonar con respuesta rápida a la misma. Fue trasladada a la unidad de cuidado intensivo, donde se inicia reanimación con líquidos endovenosos, ingresa intubada, se conecta a ventilación mecánica, se inicia tratamiento antibiótico con meropenem y vancomicina, más infusión de oxitocina. Por la hipotensión severa $(80 / 50 \mathrm{~mm} / \mathrm{Hg})$ persistente se inicia soporte vasoactivo con norepinefrina e hidrocortisona, y se adiciona claritromicina y oseltamivir al manejo. Dos días después, a pesar del manejo instaurado, la evolución de la paciente es tórpida, con hipotensión persistente dependiente de norepinefrina y con disfunción orgánica manifestada con oliguria de $0,3 \mathrm{cc} / \mathrm{kg} /$ hora, creatinina de $1,9 \mathrm{mg} / \mathrm{dL}$, recuento de plaquetas de $25.000 / \mathrm{mm}^{3}$, aminotransferasa de $53 \mathrm{UI} / \mathrm{L}$ y alaninotransferasa de $24 \mathrm{UI} / \mathrm{L}$; por todo esto, se decide como último recurso iniciar inmunomodulación con inmunoglobulina G, enriquecida con inmunoglobulina $\mathrm{M}$, la cual se inicia a dosis de $5 \mathrm{ml} / \mathrm{kg}$ /día por 3 días.

A las 24 horas después del inicio de la inmunoglobulina, las cifras tensionales y la saturación venosa central de oxígeno tienden a la estabilidad, la fiebre comienza a descender, la PVC se mantienen entre 8 y 12 , la diuresis mejora entre 1-1,5 cc/ kg/hora. Los cultivos de sangre y cordón umbilical reportan un E. Coli multisensible a los antibióticos que estaba recibiendo. Se continúa tratamiento con mejoría de la paciente. Nueve días después la paciente es extubada debido a la estabilidad hemodinámica, cardiocirculatoria, respiratoria, y de su estado ácido-base con ácido láctico de 1,6 mmol/L. Posteriormente, se traslada a sala de hospitalización con mejoría progresiva clínica y paraclínicamente. Al día 13 de hospitalización la paciente egresa de la institución en buenas condiciones generales.

\section{MATERIALES Y MÉTODOS}

Se realizó una búsqueda de la literatura médica, la cual se limitó a los idiomas inglés, portugués y español en la bases de datos de Medline via PubMed, Direct Science, Biblioteca Cochrane y SciELO, incluyendo artículos tales como metaanálisis, ensayos clínicos controlados, reportes de caso y guías de atención. Se utilizaron las palabras clave: "intravenous inmunoglobulin therapy" and "pregnancy and sepsis", e "intravenous inmunoglobulin therapy" and "obstetric sepsis".

Aspectos éticos. Se solicitó la autorización de la institución para el reporte del caso; al egreso la paciente firmó el consentimiento informado autorizando la divulgación científica de su caso. Se garantizó la confidencialidad de la información, protegiendo la identidad de la paciente y preservando sus derechos.

\section{RESULTADOS}

Se encontraron 6 artículos relacionados directamente con el uso de la inmunoglobulina en el embarazo, 1 artículo de revisión (20), 2 reportes de caso $(21,22)$ y tres guías de diagnóstico y manejo $(14,23,24)$.

Recientemente Anderson, en su artículo de revisión sobre sepsis puerperal, reporta que los estudios sobre el uso de IVIG en adultos con shock séptico no son concluyentes, pero tampoco hay estudios realizados solo en gestantes o puérperas con esta condición. Según Anderson, el shock séptico causado por estreptococos podría ser la excepción en el uso de IVIG, aunque concluye que no se cuenta con ensayos clínicos de alta calidad que puedan demostrar su utilidad (20).

Por otro lado, Izdes registra el caso de una mujer en Ankara (Turquía), de 21 años, con una gestación de 29 semanas y una sepsis causada por un acinetobacter resistente, después de una infección H1N1, quien a pesar del tratamiento, en los siguientes siete días su condición se deteriora y deciden iniciar IVIG enriquecida con IGM $20 \mathrm{mg} /$ 
$\mathrm{kg} / \mathrm{h}$ por 3,5 horas y luego $10 \mathrm{mg} / \mathrm{kg} / \mathrm{h}$ por 68 horas; la paciente gradualmente empieza a recuperarse, no se describen reacciones al medicamento. El autor considera que el uso de IVIG fue importante para la recuperación de la paciente (21).

Entre otros estudios, Al Rawi reporta el caso en el Reino Unido de una paciente de 25 años, quien 12 horas después de un parto pretérmino de 32 semanas desarrolla sepsis por estreptococo beta-hemolítico y es llevada a la UCI; debido a la poca respuesta al tratamiento, se le inicia como terapia adyuvante proteína C activada e IVIG, no se especifica la dosis ni el tiempo de esta terapia, pero reportan que al segundo día la condición de la paciente comienza a estabilizarse, no se registran efectos adversos de estos medicamentos y concluyen que el uso temprano de IVIG y proteína C activada puede conducir a buenos resultados en la sepsis materna (22).

Respecto al uso de IVIG en sepsis en obstetricia, la Guía para la atención de sepsis en el embarazo del Royal College del Reino Unido, y la Guía australiana de sepsis en el embarazo consideran que estas pueden ser útiles como terapia alternativa en shock séptico provocado por estafilococos y estreptococos recomendando su uso en estos casos, pero reportan que tienen poca evidencia de su utilidad en sepsis por Gram negativos y describen la dosis recomendada $(14,23)$. El caso presentado iría en contra de esa consideración.

Adicionalmente, la Guía para atención de las sepsis grave en paciente obstétrica de SánchezPadrón refiere que por el mecanismo de acción de las IVIG se propone su uso en sepsis, y que las IVIG se utilizan en sepsis graves por estafilococos y estreptococos, a pesar de que hay estudios en adultos críticamente enfermos que están a favor y otros en contra de su utilidad. En su guía, SánchezPadrón plantea que se cree que los resultados son mejores cuando se aplican dosis superiores a $1 \mathrm{~g} / \mathrm{kg}$, y que la IVIG rica en IgM podría tener mejores resultados que la IVIG policlonal; la dosis que recomienda es $100-200 \mathrm{mg} / \mathrm{kg} /$ día por 5 días, con un nivel 2C de recomendación según la evidencia (24). Sin embargo, el beneficio de esta recomendación no ha sido evaluado.

\section{CONCLUSIÓN}

La utilización de inmunoglobulinas intravenosas surge como una alternativa, como terapia coadyuvante, en el manejo del choque séptico en obstetricia que no responde al tratamiento convencional. Se necesitan estudios analíticos que incluyan pacientes gestantes que puedan demostrar su utilidad.

\section{REFERENCIAS}

1. American College of Chest Physicians/Society of Critical Care Medicine Consensus Conference: definitions for sepsis and organ failure and guidelines for the use of innovative therapies in sepsis. Crit Care Med. 1992;20:864-74.

2. Levy MM, Fink MP, Marshall JC, Abraham E, Angus D, Cook D, et al; 2001 SCCM/ESICM/ACCP/ATS/SIS International Sepsis Definitions Conference. Intensive Care Med. 2003;29:530-8.

3. Gómez JC. Actualización del marco conceptual y manejo de la sepsis, sepsis severa y shock séptico. Rev Med Fac. 2009;17:116-29.

4. Bamfo JE. Managing the risks of sepsis in pregnancy. Best Pract Res Clin Obstet Gynaecol. 2013;27:58395. doi: 10.1016/j.bpobgyn.2013.04.003.

5. Kramer HM, Schutte JM, Zwart JJ, Schuitemaker NW, Steegers EA, et al. Maternal mortality and severe morbidity from sepsis in the Netherlands. Acta Obstet Gynecol Scand. 2009;88:647-53.

6. Say L, Chou D, Gemmill A, Tunçalp Ö, Moller $\mathrm{AB}$, Daniels J, et al. Global causes of maternal death: a WHO systematic analysis. Lancet Glob Health. 2014;2:e323-33. doi: 10.1016/S2214109X(14)70227-X

7. Cordioli RL, Cordioli E, Negrini R, Silva E. Sepsis and pregnancy: do we know how to treat this situation? Rev Bras Ter Inten. 2013;25:334-44.

8. Kassebaum NJ, Bertozzi-Villa A, Coggeshall MS, Shackelford KA, Steiner C, Heuton KR, et al. Global, regional, and national levels and causes of maternal mortality during 1990-2013: a systematic analysis for the Global Burden of Disease Study 2013. 
Lancet. 2014;384:980-1004. doi: 10.1016/S01406736(14)60696-6.

9. Alcaldía Mayor de Bogotá D.C. Guía de sepsis en obstetricia - Secretaría Distrital de Salud. Bogotá. 2013 [visitado 2016 Ago 1]. Disponible en. http://www. saludcapital.gov.co/DDS/Publicaciones/Guia \%20 Maternidad-Sepsis_baja.pdf

10. Sung E, George J, Porter M. Sepsis in pregnancy. Fetal and Maternal Medicine Review. 2011;22:287-305.

11. Ooe K, Udagawa H. A new type of fulminant group A streptococcal infection in obstetric patients; report of 2 cases. Hum Pathol. 1997;28:509-12.

12. Snyder CC, Barton JR, Habli M, Sibai BM. Severe sepsis and septic shock in pregnancy: Indications for delivery and maternal and perinatal. J Matern Fetal Neonatal Med. 2013;26:503-6. doi: 10.3109/14767058.2012.739221.

13. Barañao RI. Inmunología del embarazo. Invest Clin. 2011;52:175-94.

14. Royal College of Obstetricians and Gynaecologists. Bacterial sepsis in pregnancy. Green-top Guideline, No. 64; 2012.

15. Dellinger RP, Levy MM, Rhodes A, Annane D, Gerlach H, Opal SM, et al. Surviving sepsis campaign: International guidelines for management of severe sepsis and septic shock: 2012. Crit Care Med. 2013;41:583637. doi: 10.1097/CCM.0b013e31827e83af.

16. Norbby-Teglund A, Haque KN, Hammarstrom L. Intravenous polyclonal IgM-enriched immunoglobulin therapy in sepsis: A review of clinical efficacy in relation to microbiological aetiology and severity of sepsis. J Intern Med. 2006;260:509-16.

17. Alejandria MM, Lansang MAD, Dans LF, Mantaring JB 3rd. Intravenous immunoglobulin for treating sepsis, severe sepsis and septic shock. Cochrane Database Syst Rev. 2013;(9):CD001090. doi: 10.1002/14651858. CD001090.pub2.

18. Loza Vázquez A, León Gil C, León Regidor A. Nuevas alternativas terapéuticas para la sepsis grave en el paciente crítico. Revisión Med Inten. 2011;35:23645. doi: 10.1016/j.medin.2010.10.002.

19. Hamano N, Nishi K, Onose A, Okamoto A, Umegaki T, Yamazaki E, et al. Efficacy of single-dose intravenous immunoglobulin administration for severe sepsis and septic shock. J Intensive Care. 2013;1:4. doi: 10.1186/2052-0492-1-4. eCollection 2013.

20. Anderson BL. Puerperal group A streptococcal infection: Beyond semmelweis. Obstet Gynecol. 2014;123:87482. doi:1097/AOG.0000000000000175.

21. Izdes S, Altintas ND, Eldem A, Ceyhan H, Kanbak O. Intravenous polyclonal IgM-enriched immunoglobulin therapy for resistant Acinetobacter sepsis in a pregnant patient with ARDS due to $\mathrm{H} 1 \mathrm{~N} 1$ infection. Int J Obstet Anesth. 2011;20:99-100. doi: 10.1016/j. ijoa.2010.09.001.

22. Al-Rawi S, Woodward LJ, Knight J. Puerperal streptococcal toxic shock syndrome treated with recombinant human activated protein $\mathrm{C}$ and intravenous immunoglobulin. Int J Obstet Anesth. 2009;18:169-72. doi: 10.1016/j.ijoa.2008.

23. South Australian Perinatal Practice Guidelines. Sepsis in pregnancy; 2014.

24. Sánchez Padron A, Sánchez Valdivia A, Somoza García M, Pérez Sánchez L. La sepsis grave en la paciente obstétrica. Guías Clínicas Breves; 2012. 